\title{
BMJ Open Sodium intake and blood pressure in children and adolescents: protocol for a systematic review and meta-analysis
}

\author{
Magali Leyvraz, ${ }^{1}$ Patrick Taffé, ${ }^{1}$ Angeline Chatelan, ${ }^{1}$ Gilles Paradis, ${ }^{2}$ René Tabin, ${ }^{3,4}$ \\ Pascal Bovet, ${ }^{1}$ Murielle Bochud, ${ }^{1}$ Arnaud Chiolero ${ }^{1,2}$
}

To cite: Leyvraz M, Taffé $P$, Chatelan A, et al. Sodium intake and blood pressure in children and adolescents: protocol for a systematic review and meta-analysis. BMJ Open 2016;6:e012518. doi:10.1136/bmjopen-2016012518

- Prepublication history and additional material is available. To view please visit the journal (http://dx.doi.org/ 10.1136/bmjopen-2016012518).

Received 3 May 2016

Revised 6 July 2016

Accepted 11 July 2016

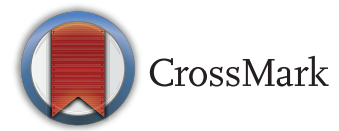

${ }^{1}$ Institute of Social and Preventive Medicine (IUMSP), Lausanne University Hospital (CHUV), Lausanne,

Switzerland

${ }^{2}$ Department of

Epidemiology, Biostatistics and Occupational Health, McGill University, Montréal, Canada

${ }^{3}$ Department of Pediatrics, Hospital of Valais, Sion, Switzerland

${ }^{4}$ Faculty of Medicine, University of Geneva, Geneva, Switzerland

Correspondence to

Magali Leyvraz;

magali.leyvraz@chuv.ch

\section{ABSTRACT}

Introduction: Hypertension is a major risk factor for cardiovascular diseases. In adults, high sodium intake is associated with elevated blood pressure. In children, experimental studies have shown that reducing sodium intake can reduce blood pressure. However, their external validity is limited, notably because the sodium reduction was substantial and not applicable in a real-life setting. Observational studies, on the other hand, allow assess the association between blood pressure and sodium intake across usual levels of consumption. There is also evidence that the association differs between subgroups of children according to age and body weight. Our objective is to conduct a systematic review and metaanalysis of experimental and observational studies on the association between sodium intake and blood pressure in children and adolescents and to assess whether the association differs according to age and body weight.

Methods and analysis: A systematic search of the MEDLINE, EMBASE, CINAHL and CENTRAL databases will be conducted and supplemented by a manual search of bibliographies and unpublished studies. Experimental and observational studies involving children or adolescents between 0 and 18 years of age will be included. The exposure will be dietary sodium intake, estimated using different methods including urinary sodium excretion. The outcomes will be systolic and diastolic blood pressure, elevated blood pressure and hypertension. If appropriate, metaanalyses will be performed by pooling data across all studies together and separately for experimental and observational studies. Subgroup meta-analyses by age and body weight will be also conducted. Moreover, separate meta-analyses for different sodium intake levels will be conducted to investigate the doseresponse relationship.

Ethics and dissemination: This systematic review and meta-analysis will be published in a peer-reviewed journal. A report will be prepared for national authorities and other stakeholders in the domains of nutrition, public health, and child health in Switzerland.

Trial registration number: CRD42016038245.

\section{Strengths and limitations of this study}

- This systematic review and meta-analysis will contribute to strengthen our knowledge on the effect of sodium intake on blood pressure in children and adolescents.

- It will be the first systematic review on this subject to include observational studies in addition to experimental studies.

- We will carry out a critical review of the methodology of the studies included.

- We will investigate whether the association between sodium intake and blood pressure in children differs according to age and body weight. Separate meta-analyses for different sodium intake levels will be also conducted to investigate the dose-response relationship.

- This protocol has been written following the Preferred Reporting Items for Systematic review and Meta-Analysis Protocols (PRISMA-P) guidelines and has been registered in PROSPERO.

\section{INTRODUCTION}

\section{Rationale}

Hypertension is a major modifiable risk factor for cardiovascular diseases and a major cause of morbidity and mortality worldwide. ${ }^{1-3}$ There is also growing evidence that elevated blood pressure has its roots early in life and that blood pressure tracks from childhood to adulthood. ${ }^{4-7}$ Further, intermediate markers of hypertensive target organ damage, such as left ventricular hypertrophy and thickening of the carotid artery vessel wall, have been found in children and adolescents with elevated blood pressure. ${ }^{6} 7$ This suggests that early life interventions aiming at the primordial prevention of elevated blood pressure could help prevent lifelong hypertension and its associated consequences. $^{89}$

Numerous studies indicate that high sodium intake is a cause of elevated blood 
pressure in adults. ${ }^{10}{ }^{11}$ For children and adolescents, much less evidence is available and findings are equivocal. Hence, while some observational studies have shown a positive association between high sodium intake and elevated blood pressure in children, ${ }^{12-16}$ others have not. ${ }^{17-20}$ Meta-analyses of experimental studies concluded that the reduction of sodium intake can lower blood pressure in children. ${ }^{21}{ }^{22}$ Their external validity is, however, limited because the sodium reduction in these trials was often substantial and likely not applicable in a real-life setting, where only modest reductions are feasible on the long run. Based on the results of experimental studies, it is therefore difficult to make recommendations applicable to the whole population. There is also evidence that some groups of children have different salt sensitivity for blood pressure depending notably on age, birth weight, prematurity or body weight. ${ }^{12}{ }^{23}$ The heterogeneity in the effect of sodium intake on blood pressure in subgroups of children has not been considered in previous reviews and meta-analyses.

Including observational studies has been recommended to overcome some limitations of meta-analyses of experimental studies, notably to increase external validity. ${ }^{24}$ Furthermore, data from observational studies would permit the assessment of the association between blood pressure and sodium intake across usual levels of consumption, as well as the determination of the shape of the association. This would also permit accounting for differences in salt sensitivity of blood pressure between various subgroups of children, in particular, according to age and body weight.

\section{Objectives}

Our primary objective is to conduct a systematic review and meta-analysis of experimental and observational studies assessing the association between sodium intake and blood pressure in children and adolescents. Our secondary objective is to assess whether this association differs according to age and body weight.

\section{METHODS AND ANALYSIS}

The protocol has been developed following the PRISMA statement for preferred reporting items for systematic reviews and meta-analysis protocols, ${ }^{25-27}$ the Meta-analysis Of Observational Studies in Epidemiology (MOOSE) guidelines ${ }^{28}$ and the Cochrane handbook for Systematic Reviews of Interventions. ${ }^{29}$ This systematic review has been registered with the International Prospective Register of Systematic Reviews (PROSPERO) (registration number CRD42016038245).

\section{Eligibility criteria}

Study designs

This systematic review will include experimental and observational studies. The following study designs will be included: randomised and non-randomised controlled and non-controlled trials, clinical trials, quasi-experimental studies, case-control, cohort and cross-sectional studies. Ecological studies, case series, case reports, reviews, meta-analyses, policy papers, comments, congress proceedings and animal studies will not be included.

\section{Participants}

Studies involving children or adolescents between 0 and 18 years of age, irrespective of sex, weight and hypertension status, will be included. Studies involving children and adults will be included only if the data for children can be extracted separately.

\section{Interventions and exposure}

For experimental studies, the intervention will be change in sodium intake. For observational studies, the exposure of interest will be the level of sodium intake. Methods of measurement of sodium intake will include dietary assessment of sodium intake (based on, eg, 24-hour recall or semi-quantitative food frequency questionnaires) and urinary sodium excretion (based on 24-hour urine collection or spot urine samples). Experimental studies with a pharmacologic intervention (ie, antihypertensive drugs or other related medication) will be excluded.

\section{Outcome measures}

The outcomes will be systolic and diastolic blood pressure, elevated blood pressure and hypertension.

\section{Timing}

There will be no restriction by duration of intervention or by length of follow-up.

\section{Setting and language}

There will be no restriction by type of setting. Studies in English, French, German or Spanish will be included.

\section{Search strategy}

A systematic search of the following electronic databases will be conducted:

- Medical Literature Analysis and Retrieval System Online (MEDLINE) by the USA National Library of Medicine (NLM) via PubMed from 1946 to present;

- Cumulative Index to Nursing and Allied Health Literature (CINAHL) via EBSCO from 1961 to present;

- Excerpta Medica database (EMBASE) by Elsevier from 1947 to present;

- Cochrane Central Register of Controlled Trials (CENTRAL) from 1974 to present.

The search strategies were developed with the help of an experienced librarian. The search strategy for MEDLINE was developed first (see box 1) and then adapted for CINAHL, EMBASE and CENTRAL (see online supplementary material files for the detailed search strategies for each databases). Three concepts 
Box 1 Search strategy for MEDLINE (MeSH, Medical Subject Headings)

1. baby[Text Word]

2. neonat $^{\star}[$ Text Word]

3. newborn*[Text Word] OR "infant, newborn"[MeSH Terms]

4. infan*[Text Word] OR infant[MeSH Terms]

5. toddler*[Text Word]

6. child ${ }^{\star}[$ Text Word] OR child[MeSH Terms]

7. preschool[Text Word] OR "child, preschool"[MeSH Terms]

8. school[Text Word]

9. pupil $^{\star}[$ Text Word] OR pupil[MeSH Terms]

10. student ${ }^{\star}[$ Text Word]

11. boy*[Text Word]

12. girl $^{\star}[$ Text Word $]$

13. adolescen*"[Text Word] OR adolescent[MeSH Terms]

14. teenage ${ }^{\star}[$ Text Word]

15. 1 OR 2 OR 3 OR 4 OR 5 OR 6 OR 7 OR 8 OR 9 OR 10 OR 11 OR 12 OR 13 OR 14

16. $\operatorname{diet}^{\star}\left[\right.$ Text Word] $O R$ diet[MeSH Terms] OR intake ${ }^{\star}[$ Text Word] OR consum*[Text Word] OR restric*[Text Word] OR reduc ${ }^{\star}[$ Text Word]

17. salt[Text Word] AND 16

18. sodium[Text Word] AND 16

19. "sodium, dietary"[MeSH Terms]

20. "sodium chloride, dietary"[MeSH Terms]

21. "diet, sodium restricted"[MeSH Terms]

22. "urinary sodium excretion"[Text Word]

23. "sodium/urine *"[MeSH Terms]

24. 17 OR 18 OR 19 OR 20 OR 21 OR 22 OR 23

25. "blood pressure"[Text Word] OR "blood pressure"[MeSH Terms]

26. "diastolic"[Text Word]

27. "systolic"[Text Word]

28. "arterial"[Text Word] OR "arterial pressure"[MeSH Terms]

29. hypertension[Text Word] OR hypertension[MeSH Terms]

30. 25 OR 26 OR 27 OR 28 OR 29

31. 15 AND 24 AND 30

were defined to determine the key terms of the search and their related terms: (1) children (related terms: baby, neonate, newborn, infant, toddler, preschool child, school child, pupil, student, boy, girl, adolescent, teenager), (2) sodium intake (related terms: salt, sodium chloride, diet, consumption, restriction, reduction, urinary sodium excretion, sodium in urine) and (3) blood pressure (related terms: arterial pressure, systolic pressure, diastolic pressure, hypertension, high blood pressure, elevated blood pressure). The database searches will use text terms and indexing terms from the thesaurus of the databases (ie, Medical Subject Headings (MeSH) for MEDLINE, CINAHL, and CENTRAL, and Emtree for EMBASE), Boolean operators and truncations.

In addition to the database search, a manual search of all the bibliographies of the retrieved articles and other reviews on the same topic will be conducted to identify additional studies. The literature search will be supplemented by a search in Web of Science and Google
Scholar. Moreover, the following clinical trial registers will be searched to identify studies of interest not yet published: International Clinical Trials Registry Platform registry (http://www.isrctn.com); the USA' ClinicalTrials. gov (http://www.clinicaltrials.gov) and the EU Clinical Trials Register (http://www.clinicaltrialsregister.eu). To access the results from completed trials found in these trial registers which do not have published reports, the principal investigators of the studies of interest will be contacted by email. A maximum of three email attempts will be conducted, after which efforts to access results will be abandoned.

\section{Study selection}

After the collection of all the potential articles, duplicates will be removed. If multiple publications are found to originate from one single study, the results of that study will be collated into one.

All the articles retrieved will be examined by two independent reviewers (ML and ACha). As a first step, each reviewer will screen the titles and abstracts and retain articles following the eligibility criteria mentioned above. As a second step, each reviewer will further assess the eligibility of each article retained after the first step by reviewing the full text article. A detailed record of the reasons for excluding studies will be kept. A data management software, Covidence (Covidence systematic review software, Veritas Health Innovation, Melbourne, Australia; www.covidence.org), will be used to organise the review and selection of articles.

Any disagreement on the exclusion or inclusion of an article between the two reviewers will be resolved by discussion or, if necessary, by a third reviewer (AChi).

\section{Data extraction}

The two reviewers will independently extract data from each included study using Microsoft Office Excel 2007. The following information will be extracted:

1. Study identification: authors, year of publication, journal, funding or sponsorship;

2. Study characteristics: country where the study was conducted, year when study was conducted, study type (observational or experimental), study design (randomised controlled study, controlled study, cohort study, cross-sectional study, etc), sampling method, sample size (total, per group and at different time points), study setting, study duration or follow-up period, participation rates and any other study characteristics;

3. Population characteristics: age, sex, weight, height, body mass index (BMI), blood pressure, hypertension status and treatment, ethnicity and other characteristics (eg, birth weight, prematurity, diabetes);

4. Exposure or intervention: for all studies, sodium intake for experimental studies, detailed information on the type and duration of the intervention will be recorded (eg, education, individual counselling, 
cooking demonstrations, reduction of foods with high sodium, low sodium bread, low sodium drinking water, low sodium school meals, low sodium infant formula, diet high in fruits and vegetables, etc);

5. Sodium intake measurement method: dietary intake assessment method (eg, 24-hour recall or semiquantitative food frequency questionnaire, validated questionnaire or not), urine collection method (eg, spot test, single or repeated 24-hour urine collection), number of measurements, training of persons doing the measurement, etc;

6. Outcome: systolic and diastolic blood pressure, elevated blood pressure and hypertension;

7. Outcome measurement method: that is, auscultatory versus oscillometry, type of blood pressure measurement device, office versus home versus ambulatory measurement, number of visits, number of measurements per visit, training of persons doing the measurements, use of a standardised protocol, cuff size, etc;

8. Potential confounding and effect modification factors: if available, information on potential confounding factors, for example, body weight, potassium and calcium intakes, and potential effect modification factors, for example, age, body weight, sex and ethnicity, will be recorded. Any adjustment for these variables will be documented and considered when conducting the meta-analyses.

\section{Study quality}

Information on the data quality will be extracted for all studies according to the four criteria outlined in table 1. Further, for experimental studies, an additional assessment of the data quality will be done according to the Cochrane collaboration's risk of bias tool..$^{29}$ For observational studies, the data quality will also be assessed according to the Newcastle-Ottawa Scale for nonrandomised studies. ${ }^{30}$

If essential information on the study is missing, the corresponding author of the article will be contacted by email to obtain the missing information (maximum of three email attempts). Any disagreement on the extracted data and data quality assessments between the two reviewers will be resolved by discussion or, if necessary, with a third reviewer.

\section{Data analyses}

The statistical analyses will be conducted with RStudio (V.0.99.473), Stata (V.14.1) and RevMan (V.5.3) (Review Manager (RevMan) [Computer program]. 5.3 version. Copenhagen: The Nordic Cochrane Centre, 2014).

\section{Measures of effect or association}

For the experimental studies, the measure of effect for continuous outcomes will be defined as the difference in means of blood pressure levels between the intervention and the control group. For dichotomous outcomes, that is, elevated blood pressure and hypertension, the measure of effect will be the OR or the relative risk. If data are available at multiple time points, the time points nearest to the beginning and the end of the intervention will be selected.

For the observational studies, the measure of association for continuous outcomes will be the correlation (or regression coefficient) between sodium intake and blood pressure. For dichotomous outcomes, the OR at different sodium intake ranges will be used as the main measure of association.

The different measures of exposure and outcome, and the corresponding measures of effect and association, will be converted into sodium intakes in grams per day,

\begin{tabular}{|c|c|}
\hline $\begin{array}{l}\text { Quality } \\
\text { level }\end{array}$ & Criteria \\
\hline \multicolumn{2}{|c|}{ Method for exposure measurement } \\
\hline High & Urinary sodium excretion by 24 -hour urine collection and high quality of urine collection \\
\hline Low & Urine spot test or questionnaire \\
\hline Unclear & Insufficient information to permit judgement \\
\hline \multicolumn{2}{|c|}{ Method and setting for measurement of outcome } \\
\hline High & $\begin{array}{l}\text { Measured multiple times, by trained professional and using standardised procedures. If measured by } \\
\text { oscillometric method, the device is clinically validated. }\end{array}$ \\
\hline Low & Otherwise (if not all criteria mentioned above are met) \\
\hline Unclear & Insufficient information to permit judgement \\
\hline \multicolumn{2}{|c|}{ External validity } \\
\hline High & Characteristics of study population are relatively representative of, and generalizable to, the general population \\
\hline Low & $\begin{array}{l}\text { Characteristics of study population are present in only a specific part of the population (eg, hypertensive } \\
\text { children, obese children, etc) }\end{array}$ \\
\hline Unclear & Insufficient information to permit judgement \\
\hline \multicolumn{2}{|l|}{ Reporting } \\
\hline High & Complete report and if applicable trial registration \\
\hline Low & $\begin{array}{l}\text { Incomplete report (eg, not all prespecific primary outcomes are reported), source of financial support is missing } \\
\text { or impartial }\end{array}$ \\
\hline Unclear & Insufficient information to permit judgement \\
\hline
\end{tabular}


systolic and diastolic blood pressure levels in $\mathrm{mm} \mathrm{Hg}$, and prevalence of elevated blood pressure and hypertension in percentage. If the sodium intake is available in ranges only, the midpoint of the range will be taken.

\section{Meta-analyses}

It is anticipated that there will be a sufficient number of studies to conduct meta-analyses. In brief, meta-analyses will be performed by pooling data across all studies together and separately for experimental and observational studies. Subgroup meta-analyses by age and body weight will be also conducted to evaluate whether the association between sodium intake and blood pressure differs (1) between younger and older children and (2) between normal weight and overweight/obese children.

Random effect analyses will be performed, as heterogeneity in the effect size estimate between studies is expected. Forest plots will be used to present the results graphically. To better express uncertainties in the effect estimates, $95 \%$ prediction intervals will be computed. ${ }^{31} 32$ If study results are reported in a format that cannot be converted to a standard metric, they will be summarised in a narrative format.

\section{Dose-response relationship}

The relation between sodium intake and blood pressure has been hypothesised to be linear and more recently $\mathrm{j}$ shaped. ${ }^{33}$ The dose-response relationship between sodium intake and blood pressure will be investigated by conducting separate meta-analyses for incremental levels of sodium intakes. Various models, that is, linear and polynomial, will be investigated and fitted to the collected data. If sufficient data are available, this analysis will be stratified by age and body weight.

\section{Assessment of heterogeneity}

The heterogeneity will be assessed by the $\mathrm{I}^{2}$ statistic and between-study variance $\sigma^{2}$. Sources of heterogeneity will be explored using subgroup analyses and meta-regression, ${ }^{29}$ if sufficient studies are included, based on the following variables: study design, study or intervention duration, sample size, adjustments made, sodium intake ranges and measurement methods, blood pressure measurement methods, potassium intake, calcium intake, age, sex and weight. Sensitivity analyses will be performed (1) excluding relatively small studies, (2) restricting analyses to studies of high quality and (3) restricting analyses to studies using 24-hour urine collection to assess sodium intake.

\section{Assessment of publication bias}

Publication bias will be evaluated by enhanced funnel plots and Egger's test. ${ }^{29} 34$

\section{Assessment of strength of evidence}

The strength of the body of evidence for this systematic review (ie, for the ensemble of all the studies included in the review) will be assessed using The Grading of Recommendations, Assessment, Development and Evaluation (GRADE) framework. ${ }^{35}$

\section{ETHICS AND DISSEMINATION}

The results of this systematic review will summarise the current state of evidence for the association between sodium intake and blood pressure in children. The results of this systematic review will be critically interpreted and discussed in light of the quality of the studies included. Moreover, it will broaden the results of previous reviews, notably by the potential identification of subgroups of children highly sensitive to sodium intake and for which targeted intervention aiming at reducing of sodium intake could be justified.

This systematic review will be reported according to the recommendations of the PRISMA statement. ${ }^{27}$ The results will be disseminated via the publication of one or more manuscripts in a peer-reviewed journal and the presentation at one or more conferences. Moreover, a report will be prepared for the Swiss Federal Food Safety and Veterinary Office and for other stakeholders in the domains of nutrition, public health and child health in Switzerland. This report will present the results and will discuss the implications of the findings in general and more specifically for Switzerland.

Acknowledgements The authors thank Thomas Brauchli for his help on developing the search strategy.

Contributors ML and AChi prepared the research protocol and drafted the manuscript. PT carefully reviewed the statistical analyses. ACha, GP, RT, PB and MB made substantial contributions to the manuscript. All authors read and approved the final version of the manuscript.

Funding This systematic review is funded by the Swiss Federal Food Safety and Veterinary Office (FSVO) (funding reference number 5.15.03). The funder will have no involvement in the protocol design, the execution of the systematic review and meta-analysis, the data analyses, the interpretation or the publication of the results.

Competing interests None declared.

Provenance and peer review Not commissioned; externally peer reviewed.

Data sharing statement All data will be published as part of the systematic review. The data set supporting the conclusions of this article will be made available in the DATA@IUMSP repository, http://data.iumsp.ch.

Open Access This is an Open Access article distributed in accordance with the Creative Commons Attribution Non Commercial (CC BY-NC 4.0) license, which permits others to distribute, remix, adapt, build upon this work noncommercially, and license their derivative works on different terms, provided the original work is properly cited and the use is non-commercial. See: http:// creativecommons.org/licenses/by-nc/4.0/

\section{REFERENCES}

1. Lawes CM, Vander Hoorn S, Rodgers A, et al. Global burden of blood-pressure-related disease, 2001. Lancet 2008;371: 1513-18.

2. Bochud $M$, Marques-Vidal $P$, Burnier $M$, et al. Dietary salt intake and cardiovascular disease: summarizing the evidence. Public Health Rev 2012;33:530-52.

3. Forouzanfar MH, Alexander L, Anderson HR, et al. Collaborators GBDRF. Global, regional, and national comparative risk assessment of 79 behavioural, environmental and occupational, and metabolic risks or clusters of risks in 188 countries, 1990-2013: a systematic analysis for the Global Burden of Disease Study 2013. Lancet 2015;386:2287-323.

4. Chen $X$, Wang $Y$. Tracking of blood pressure from childhood to adulthood: a systematic review and meta-regression analysis. Circulation 2008;117:3171-80. 
5. Chiolero A, Cachat F, Burnier M, et al. Prevalence of hypertension in schoolchildren based on repeated measurements and association with overweight. J Hypertens 2007;25:2209-17.

6. McCrindle BW. Assessment and management of hypertension in children and adolescents. Nat Rev Cardiol 2010;7:155-63.

7. Chiolero A, Bovet P, Paradis G. Screening for elevated blood pressure in children and adolescents: a critical appraisal. JAMA Pediatr 2013;167:266-73.

8. Labarthe DR. Prevention of cardiovascular risk factors in the first place. Prev Med 1999;29(Pt 2):S72-8.

9. Gillman MW. Primordial prevention of cardiovascular disease. Circulation 2015;131:599-601.

10. WHO. Reducing salt intake in populations: report of a WHO forum and technical meeting. 5-7 October 2006, Paris, France. Paris: World Health Organization, 2006.

11. He FJ, Li J, Macgregor GA. Effect of longer term modest salt reduction on blood pressure: Cochrane systematic review and meta-analysis of randomised trials. BMJ 2013;346:f1325.

12. Yang Q, Zhang Z, Kuklina EV, et al. Sodium intake and blood pressure among US children and adolescents. Pediatrics 2012;130:611-19.

13. Cooper R, Soltero I, Liu K, et al. The association between urinary sodium excretion and blood pressure in children. Circulation 1980;62:97-104.

14. Rosner B, Cook NR, Daniels S, et al. Childhood blood pressure trends and risk factors for high blood pressure: the NHANES experience 1988-2008. Hypertension 2013;62:247-54.

15. Shi L, Krupp D, Remer T. Salt, fruit and vegetable consumption and blood pressure development: a longitudinal investigation in healthy children. Br J Nutr 2014;111:662-71.

16. Maldonado-Martín A, García-Matarín L, Gil-Extremera B, et al. Blood pressure and urinary excretion of electrolytes in Spanish schoolchildren. J Hum Hypertens 2002;16:473-8.

17. Cooper R, Liu K, Trevisan M, et al. Urinary sodium excretion and blood pressure in children: absence of a reproducible association. Hypertension 1983;5:135-9.

18. Ounaissa K, Ksira I, Ben Romdhane M, et al. P-051: relationship between blood pressure profile, and anthropometric and nutritional profiles of a population of obese children and adolescents. Ann Cardiol Angeiol (Paris) 2015;64(Suppl 1):S41.

19. Geleijnse JM, Grobbee DE, Hofman A. Sodium and potassium intake and blood pressure change in childhood. $B M J$ 1990;300:899-902.

20. Yamauchi T, Furuta M, Hamada J, et al. Dietary salt intake and blood pressure among schoolchildren. Ann Physiol Anthropol 1994:13:329-36.

21. He FJ, MacGregor GA. Importance of salt in determining blood pressure in children: meta-analysis of controlled trials. Hypertension 2006;48:861-9.
22. WHO. Effect of reduced sodium intake on blood pressures and potential adverse effects in children. Geneva: World Health Organization, 2012

23. Simonetti GD, Raio L, Surbek D, et al. Salt sensitivity of children with low birth weight. Hypertension 2008;52:625-30.

24. Shrier I, Boivin JF, Steele RJ, et al. Should meta-analyses of interventions include observational studies in addition to randomized controlled trials? A critical examination of underlying principles. Am J Epidemiol 2007;166:1203-9.

25. Liberati A, Altman DG, Tetzlaff J, et al. The PRISMA statement for reporting systematic reviews and meta-analyses of studies that evaluate healthcare interventions: explanation and elaboration. BMJ 2009;339:b2700.

26. Moher D, Shamseer L, Clarke M, et al. Preferred reporting items for systematic review and meta-analysis protocols (PRISMA-P) 2015 statement. Syst Rev 2015;4:1.

27. Shamseer L, Moher D, Clarke $M$, et al. Preferred reporting items for systematic review and meta-analysis protocols (PRISMA-P) 2015: elaboration and explanation. BMJ 2015;349:97647.

28. Stroup DF, Berlin JA, Morton SC, et al. Meta-analysis of observational studies in epidemiology: a proposal for reporting Meta-analysis Of Observational Studies in Epidemiology (MOOSE) group. JAMA 2000;283:2008-12.

29. Higgins JPT, Green S, ed. Cochrane Handbook for Systematic Reviews of Interventions. Version 5.1.0 [updated March 2011]. The Cochrane Collaboration, 2011. Available from www.handbook. cochrane.org (accessed 16 Sept 2016).

30. Wells GA, Shea B, O'Connell D, et al. The Newcastle-Ottawa Scale (NOS) for assessing the quality of nonrandomised studies in metaanalyses. Available from: http://www.ohri.ca/programs/clinical_ epidemiology/oxford.htm (accessed 16 Sept 2016).

31. Borenstein M, Hedges LV, Higgins JPT, et al. Prediction intervals. In: John Wiley \& Sons L, ed. Borenstein M, Hedges LV, Higgins JPT, Rothstein HR. Introduction to meta-analysis. Chichester, UK, 2009:127-33.

32. Chiolero A, Santschi V, Burnand B, et al. Meta-analyses: with confidence or prediction intervals? Eur J Epidemiol 2012;27:823-5.

33. Alderman $\mathrm{MH}$, Cohen HW. Dietary sodium intake and cardiovascular mortality: controversy resolved? Am J Hypertens 2012;25: 727-34

34. Egger M, Davey Smith G, Schneider M, et al. Bias in metaanalysis detected by a simple, graphical test. BMJ 1997;315: 629-34.

35. Schünemann H, Brozek J, Guyatt G, et al, eds. Handbook for grading the quality of evidence and the strength of recommendations using the GRADE approach. Updated October 2013. http://gdt. guidelinedevelopment.org/app/handbook/handbook.html (accessed 16 Sept 2016). 